\title{
Metal versus rare-gas ion irradiation during Ti1-xAlxN film growth by hybrid high power pulsed magnetron/dc magnetron co-sputtering using synchronized pulsed substrate bias
}

\author{
Grzegorz Greczynski, Jun Lu, Jens Jensen, Ivan Petrov, Joseph E. Greene, Stephan Bolz, \\ Werner Koelker, Christoph Schiffers, Oliver Lemmer and Lars Hultman
}

\section{Linköping University Post Print}

N.B.: When citing this work, cite the original article.

Original Publication:

Grzegorz Greczynski, Jun Lu, Jens Jensen, Ivan Petrov, Joseph E. Greene, Stephan Bolz, Werner Koelker, Christoph Schiffers, Oliver Lemmer and Lars Hultman, Metal versus raregas ion irradiation during Ti1-xAlxN film growth by hybrid high power pulsed magnetron/dc magnetron co-sputtering using synchronized pulsed substrate bias, 2012, Journal of Vacuum Science \&amp; Technology. A. Vacuum, Surfaces, and Films, (30), 6, 061504.

http://dx.doi.org/10.1116/1.4750485

Copyright: American Vacuum Society http://www.avs.org/

Postprint available at: Linköping University Electronic Press http://urn.kb.se/resolve?urn=urn:nbn:se:liu:diva-88465 


\title{
Metal vs. Rare-gas Ion Irradiation during $\mathrm{Ti}_{1-x} \mathrm{Al}_{x} \mathbf{N}$ Film Growth by Hybrid HIPIMS/DCMS Co-sputtering using Synchronized Pulsed Substrate Bias
}

\author{
G. Greczynski, ${ }^{1}$ J. Lu, ${ }^{1}$ J. Jensen, ${ }^{1}$ I. Petrov,${ }^{1,2}$ J.E. Greene, ${ }^{1,2,3}$ S. Bolz, ${ }^{4}$ W. Kölker, ${ }^{4}$ \\ Ch. Schiffers, ${ }^{4}$ O. Lemmer ${ }^{4}$ and L. Hultman ${ }^{1}$ \\ ${ }^{1}$ Department of Physics (IFM), Linköping University, SE-581 83 Linköping, Sweden \\ ${ }^{2}$ Materials Science Department and Frederick Seitz Materials Research Laboratory, \\ University of Illinois, Urbana, Illinois 61801 \\ ${ }^{3}$ Department of Physics, University of Illinois, Urbana, Illinois 61801, USA \\ ${ }^{4}$ CemeCon AG, Adenauerstr. 20 A4, D-52146 Würselen, Germany
}

\begin{abstract}
Metastable $\mathrm{NaCl}$-structure $\mathrm{Ti}_{1-x} \mathrm{Al}_{x} \mathrm{~N}$ is employed as a model system to probe the effects of metal vs. rare-gas ion irradiation during film growth using reactive high-power pulsed magnetron sputtering (HIPIMS) of $\mathrm{Al}$ and de magnetron sputtering (DCMS) of Ti. The alloy film composition is chosen to be $x=0.61$, near the kinetic solubility limit at the growth temperature of $500{ }^{\circ} \mathrm{C}$. Three sets of experiments are carried out: a $-60 \mathrm{~V}$ substrate bias is applied either continuously, in synchronous with the full HIPIMS pulse, or in synchronous only with the metal-rich-plasma portion of the HIPIMS pulse. Alloy films grown under continuous dc bias exhibit a thickness-invariant small-grain, two-phase nanostructure (wurtzite $\mathrm{AlN}$ and cubic $\mathrm{Ti}_{1-x} \mathrm{Al}_{x} \mathrm{~N}$ ) with random orientation, due primarily to intense $\mathrm{Ar}^{+}$irradiation leading to Ar incorporation (0.2 at\%), high compressive stress (-4.6 GPa), and material loss by resputtering. Synchronizing the bias with the full HIPIMS pulse results in films which exhibit much lower stress levels (-1.8 GPa) with no measureable $\mathrm{Ar}$ incorporation, larger grains elongated in the growth direction, a very small volume fraction of wurtzite AlN, and random orientation. By synchronizing the bias with the metal-plasma phase of the HIPIMS pulses, energetic $\mathrm{Ar}^{+}$ion bombardment is greatly reduced in favor of irradiation predominantly by $\mathrm{Al}^{+}$ions. The resulting films are single phase with a dense competitive columnar structure, strong 111 orientation, no measureable trapped Ar concentration, and even lower stress $(-0.9 \mathrm{GPa})$. Thus, switching from $\mathrm{Ar}^{+}$to $\mathrm{Al}^{+}$ bombardment, while maintaining the same integrated incident ion/metal ratio, eliminates
\end{abstract}


phase separation, minimizes renucleation during growth, and reduces the high concentration of residual point defects which give rise to compressive stress.

\section{INTRODUCTION}

Thin films of metastable $\mathrm{NaCl}$-structure $\mathrm{Ti}_{1-x} \mathrm{Al}_{x} \mathrm{~N}$ exhibiting high hardness and good high-temperature oxidation resistance ${ }^{1}$ are used for wear protection in a wide variety of applications ranging from cutting tools to mechanical components in the aerospace industry. ${ }^{2}$ Enhanced performance at elevated temperatures is obtained with single-phase alloy films having high AlN content. However, kinetic solid solubilities of wurtzite-structure AlN (wAlN) in cubic TiN depend sensitively on film growth parameters. ${ }^{3}$ At thermodynamic equilibrium, the solubility of AlN in TiN is low, only $2 \mathrm{~mol} \%$ at $1000{ }^{\circ} \mathrm{C}$ (Ref.4) and $5 \mathrm{~mol} \%$ at $2425{ }^{\circ} \mathrm{C} .{ }^{5}$ Recently, we have shown that single-phase $\mathrm{Ti}_{1-x} \mathrm{Al}_{x} \mathrm{~N}$ alloys with $x$ up to 64 mol\% can be grown using a hybrid co-sputtering technique combining high-power pulsed magnetron sputtering (HIPIMS) of $\mathrm{Al}$ and dc magnetron sputtering (DCMS) of Ti. ${ }^{6,7}$ The primary ions during Al HIPIMS are $\mathrm{Al}^{+}{ }^{7}$ Reversing the power supplies, such that the $\mathrm{Ti}$ target is powered by HIPIMS and Al sputtered in the DCMS mode, reduces $x_{\max }$ to $\sim 40$ mol\%, due to bombardment by high-energy $\mathrm{Ti}^{2+}$ ions during the Ti HIPIMS pulse. The resulting high defect densities lead to the nucleation of wurtzite-structure AlN second-phase precipitates.

The purpose of the present research is to probe the effects of synchronous biasing during film growth by hybrid HIPIMS/DCMS co-sputtering. We use metastable $\operatorname{Ti}_{0.39} \mathrm{Al}_{0.61} \mathrm{~N}$ alloy films, grown at $500{ }^{\circ} \mathrm{C}$ by Al-HIPIMS/Ti-DCMS, as a model system. A $-60 \mathrm{~V}$ bias is applied to the substrate using three different modes: (1) the bias is continuous during both HIPIMS pulses and DCMS, (2) the bias is applied in synchronous with the full HIPIMS 
pulse, and (3) the bias is applied only in synchronous with the metal-rich-plasma portion of the HIPIMS pulse.

Alloy films grown under continuous dc bias are small-grain, two-phase mixtures consisting of AlN-deficient NaCl-structure $\mathrm{Ti}_{1-x} \mathrm{Al}_{x} \mathrm{~N}$ and wurtzite-structure AlN, with high compressive stress, a high concentration of trapped Ar, and no preferred orientation. Synchronizing the bias with the full HIPIMS pulse on the Al target yields films with no measureable Ar concentration, significantly lower compressive stress, larger average grain size, a smaller volume fraction of wurtzite AlN, and random texture. By synchronizing the bias with the metal plasma phase of the HIPIMS pulses, high-energy $\mathrm{Ar}^{+}$ion irradiation is greatly reduced in favor of irradiation predominantly by $\mathrm{Al}^{+}$ions. The resulting single-phase $\mathrm{NaCl}$-structure $\mathrm{Ti}_{1-x} \mathrm{Al}_{x} \mathrm{~N}$ alloys have a dense competitive columnar structure with a strong 111 orientation and even lower stress.

\section{EXPERIMENTAL}

$\mathrm{Ti}_{0.39} \mathrm{Al}_{0.61} \mathrm{~N}$ films are grown in a $\mathrm{CC} 800 / 9$ CemeCon $\mathrm{AG}$ magnetron sputtering system $^{8}$ equipped with cast rectangular $8.8 \times 50 \mathrm{~cm}^{2} \mathrm{Ti}$ and $\mathrm{Al}$ targets. $\mathrm{Si}(001)$ substrates, $3 \times 1$ $\mathrm{cm}^{2}$, are mounted symmetrically with respect to the targets, which are tilted toward the substrate, resulting in a $21^{\circ}$ angle between the substrate normal and the normal to the target. Target-to-substrate distance is $18 \mathrm{~cm}$. The substrates are cleaned sequentially in acetone and isopropyl alcohol and mounted with clips such that their long sides are parallel to the long sides of the targets. The system is thoroughly degassed before deposition; we use two-hour long heating cycle, consisting of two steps. First, two resistive heaters are powered to $10 \mathrm{~kW}$ for 1 hour, resulting in a chamber temperature of $600{ }^{\circ} \mathrm{C}$. Following this the heating power is reduced to $7.3 \mathrm{~kW} /$ heater for 1 hour (chamber temperature $=500{ }^{\circ} \mathrm{C}$ ) and maintained at this

level during film deposition. The system base pressure is $<2.3 \times 10^{-6}$ Torr $(0.3 \mathrm{mPa})$ and the 
total pressure $P_{\text {tot }}$ during deposition is $3 \mathrm{mTorr}(0.4 \mathrm{~Pa})$. Ar flow is set at $350 \mathrm{~cm}^{3} / \mathrm{min}(\mathrm{sccm})$, while the $\mathrm{N}_{2}$ flow is controlled by a feedback loop to maintain $P_{t o t}$ constant and varies between runs from $80 \mathrm{sccm}$ to $72 \mathrm{sccm}$. The film growth temperature $T_{s}$ is $500{ }^{\circ} \mathrm{C}$.

The $\mathrm{Al}$ target is powered in HIPIMS mode $(5 \mathrm{~J} /$ pulse, $500 \mathrm{~Hz}, 10 \%$ duty cycle), while the Ti target is operated as a conventional dc magnetron at an average power $P_{d c}$ of $2.4 \mathrm{~kW}$. As-deposited alloys films have AlN concentrations of $61 \pm 1$ mol\%. The AlN deposited thickness per HIPIMS pulse $(200 \mu \mathrm{s})$ is $4.5 \times 10^{-3} \AA\left(\sim 3.7 \times 10^{-3}\right.$ monolayer, ML, for 111 crystallites), while the steady-state DCMS TiN deposition rate is $1.37 \AA$ A/s. Thus, the DCMS TiN thickness during a single HIPIMS period $(2 \mathrm{~ms})$ is $2.7 \times 10^{-3} \AA, \sim 2.2 \times 10^{-3}$ ML.

$\mathrm{Ti}_{1-x} \mathrm{Al}_{x} \mathrm{~N}$ films are grown with a substrate bias, $V_{s}=-60 \mathrm{~V}$, applied using one of three biasing schemes: (1) continuous bias; (2) bias applied synchronously during the full $200 \mu \mathrm{s}$ HIPIMS pulse (between HIPIMS pulses, the substrate is at floating potential, $V_{f} \sim-10 \mathrm{~V}$ ); or (3) bias synchronized with the $60 \mu \mathrm{s}$ metal-rich-plasma portion of the HIPIMS pulse beginning at $40 \mu \mathrm{s}$ after pulse initiation (at all other times, the substrate is at $V_{f}$ ). The total film deposition time is 90 minutes leading to film thicknesses of (1) 1.2 , (2) 1.9, and (3) 1.9 $\mu \mathrm{m}$, as determined by scanning electron microscopy (SEM) of fracture cross-sections and confirmed by cross-sectional transmission electron microscopy (XTEM).

The substrate bias voltage and current are monitored with a Tektronix DPO4054 500 MHz bandwidth four-channel digital oscilloscope.

Time-resolved measurements of ion fluxes incident at the growing film during HIPIMS sputtering of the Al target and DCMS sputtering of the Ti target are obtained using a Hiden Analytical PSM003 mass spectrometer mounted at the substrate position as in Ref. 7. For HIPIMS operation, data are collected during 50 consecutive pulses such that the total acquisition time per data point is $1 \mathrm{~ms}$. $\mathrm{Ar}, \mathrm{N}_{2}, \mathrm{~N}, \mathrm{Al}$, and Ti single and multiply charged ion energy distribution functions $\left(\mathrm{IEDF}_{\mathrm{S}}\right)$ are recorded with energy steps of $1 \mathrm{eV}$. 
$\mathrm{Ti}_{1-x} \mathrm{Al}_{x} \mathrm{~N}$ film compositions are determined to within an uncertainty of \pm 1 at $\%$ by timeof-flight elastic recoil detection analysis (ToF-ERDA) employing a $36 \mathrm{MeV}{ }^{127} \mathrm{I}^{8+}$ probe beam. X-ray diffraction (XRD), together with plan-view and cross-sectional transmission electron microscopy (TEM and XTEM), are used to analyze the nano- and microstructure of as-deposited layers, while film stress $\sigma$ is obtained from XRD $\sin ^{2} \psi$ analyses. ${ }^{9}$

\section{RESULTS}

In situ ion mass spectrometry measurements reveal the presence of high-intensity energetic $\mathrm{Al}^{+}$fluxes incident at the substrate position during HIPIMS pulses. $\mathrm{Ar}^{+}, \mathrm{Ar}^{2+}, \mathrm{N}_{2}^{+}$, and $\mathrm{N}^{+}$gas ions are also detected. The $\mathrm{Al}^{+}$ion intensity integrated over the $200-\mu$ s pulse constitutes $44.5 \%$ of the entire ion flux and is comparable to the integrated intensity of $\mathrm{Ar}^{+}$ ions, $41.8 \%$. However, the mean metal-ion energy is significantly higher, particularly during the time interval when the HIPIMS target current density $J_{T}$ is near its maximum value (see dashed curve in Fig.1). Within the $20 \mu$ s window $(t=40-60 \mu \mathrm{s})$ for which $J_{T}$ ranges from 0.41 to $0.47 \mathrm{~A} / \mathrm{cm}^{2}$, mean ion energies incident at the substrate plane are $11.4 \mathrm{eV}$ for $\mathrm{Al}^{+} v s$. $1.8 \mathrm{eV}$ for $\mathrm{Ar}^{+}$. The flux of $\mathrm{Al}^{2+}$ ions is negligible (less than $0.05 \%$ of the total ion flux to the substrate), primarily due to the relatively high second-ionization potential of $\mathrm{Al}(18.83 \mathrm{eV})^{10}$ and the short plasma transit time of the relatively low-mass sputter-ejected $\mathrm{Al}$ atoms $\left(m_{A l}=\right.$ $27 \mathrm{amu})$. Doubly-ionized Ar ions $\mathrm{Ar}^{2+}(27.63 \mathrm{eV})^{10}, 0.2 \%$ of the ion flux, have a mean energy of $10.5 \mathrm{eV} . \mathrm{N}_{2}{ }^{+}$and $\mathrm{N}^{+}$reactive gas ions are detected with relative contributions of 10.1 and $3.4 \%$, and mean energies of 3.7 and $7.7 \mathrm{eV}$, respectively.

Time-dependent intensities of the primary ion fluxes -- $\mathrm{Ar}^{+}, \mathrm{N}_{2}^{+}, \mathrm{N}^{+}$, and $\mathrm{Al}^{+}--$ incident at the growing film during Al-HIPIMS are plotted in Figure 1 with a $20 \mu \mathrm{s}$ resolution. Each data point at time $t$ represents the number of ions collected during the interval from $t-10$ to $t+10 \mu$ s. As reported previously for reactive Al-HIPIMS in $\mathrm{Ar} / \mathrm{N}_{2}$ 
mixtures, ${ }^{7}$ the plasma is initially maintained primarily by $\mathrm{Ar}^{+}$and $\mathrm{N}_{2}{ }^{+}$gas ions. The non-zero flux intensities detected at $t=0 \mu$ s in Figure 1 are due to residual ionized species from the previous pulse. The $\sim 20 \mu$ s time delay before the appearance of atomic $\mathrm{N}^{+}$ions, together with the high $\mathrm{N}^{+}$mean energy (relative to $\mathrm{N}_{2}{ }^{+}$ions), indicates that they originate primarily from $\mathrm{N}$ atoms sputter ejected from the target surface and ionized in the plasma. Gas-ion intensities begin to decrease after $30-40 \mu$ s due to rarefaction stemming from the high thermal and momentum load supplied by the HIPIMS target.

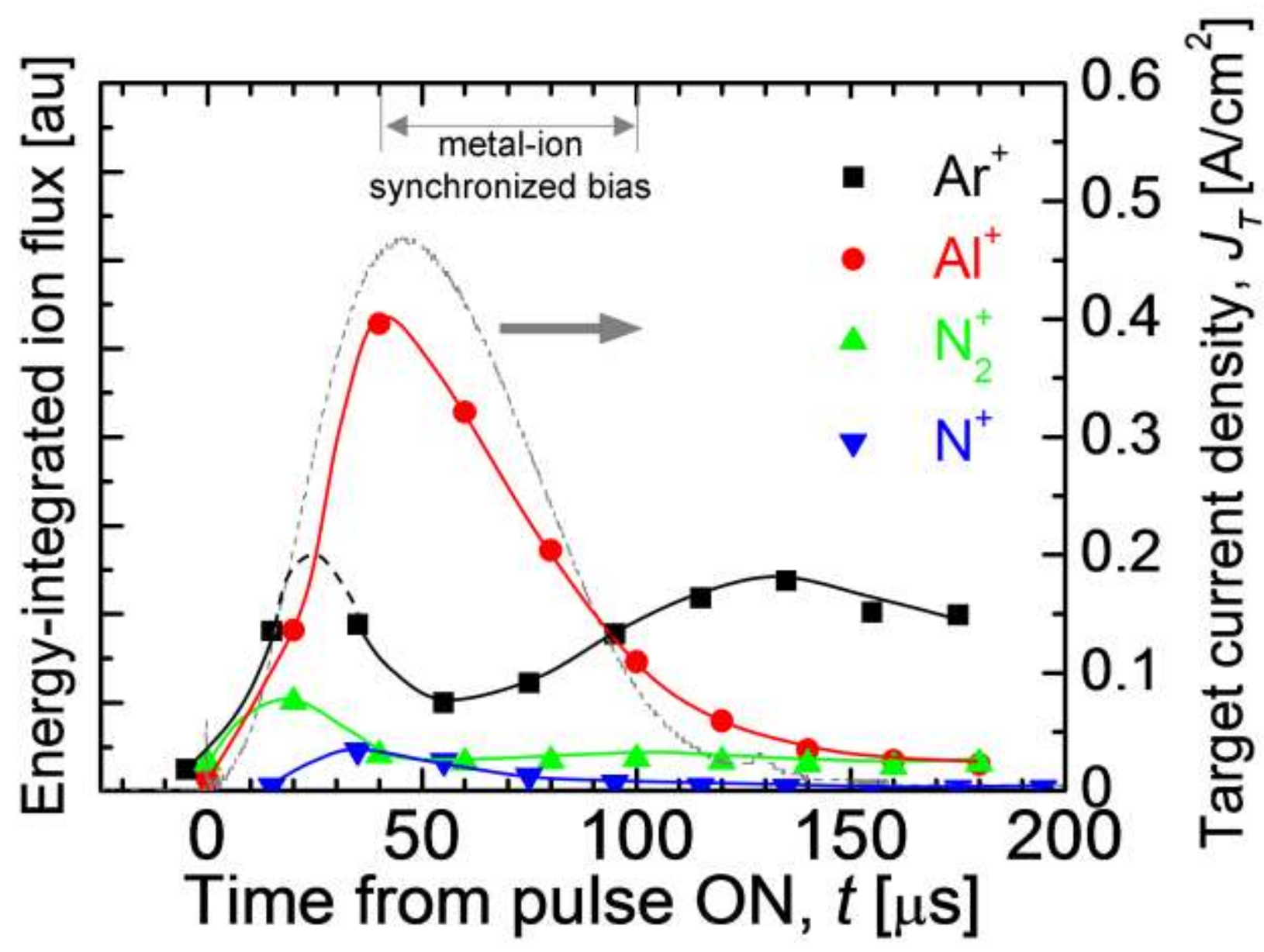

Fig.1 Time evolution of the energy-integrated flux of ions incident at the substrate: $\mathrm{Ar}^{+}$, $\mathrm{Al}^{+}, \mathrm{N}_{2}{ }^{+}$, and $\mathrm{N}^{+}$. The target current density waveform is shown for reference.

$\mathrm{Al}$ has a low first ionization energy $(5.986 \mathrm{eV})^{10}$ and $\mathrm{Al}^{+}$is the dominant plasma ion from $t \sim 40$ to $100 \mu$ s into the pulse. Electron-impact ionization of sputter-ejected Al atoms reduces the intensity of the high-energy tail in the plasma electron energy distribution. This, 
in turn, decreases the ionization probability of gas species $\mathrm{Ar}, \mathrm{N}_{2}$, and $\mathrm{N}$ which have ionization energies $15.75,15.55$, and $14.50 \mathrm{eV} .{ }^{10} \mathrm{Al}^{+}$intensity, after reaching a peak at $\sim 50$ $\mu$ s, begins to decrease due to a corresponding decrease in the discharge current as the plasma becomes power-supply limited. The $\mathrm{Ar}^{+}$intensity again dominates during the latter half of the pulse, from $t \sim 100$ to $200 \mu \mathrm{s} . \mathrm{N}_{2}{ }^{+}$and $\mathrm{N}^{+}$intensities do not fully recover due to increased target sputtering rates giving rise to efficient trapping and chemisorption of nitrogen by freshly-deposited $\mathrm{Al}$ atoms.

A comparison of the total ion flux (primarily $\mathrm{Al}^{+}$and $\mathrm{Ar}^{+}$) during a HIPIMS pulse to the continuous ion flux (dominated by $\mathrm{Ar}^{+}$) from dc magnetron sputtering is obtained from the time-averaged ion saturation current densities $J_{s}$ measured using a 15-mm-diameter circular probe mounted at the substrate position during Al-HIPIMS and Ti-DCMS operation. $J_{s}$ is $0.60 \mathrm{~mA} / \mathrm{cm}^{2}$ in HIPIMS and $0.35 \mathrm{~mA} / \mathrm{cm}^{2}$ during DCMS.

Since time-resolved ion mass spectrometry measurements in the Al-HIPIMS plasma indicate that the peak total ion count during the $200-\mu$ s HIPIMS pulse is $\sim 8 \times$ higher than the average value obtained between the pulses, we conclude that the ion flux arising from the HIPIMS source during the pulse is at least an order of magnitude higher than the concurrent ion bombardment from the Ti plasma. Therefore, film growth conditions during the highpower pulses are, to a first approximation, controlled by the HIPIMS plasma.

Throughout the time between the $200 \mu$ s HIPIMS pulses, the growing film continues to be exposed to a large $\mathrm{Ar}^{+}$ion flux from the DCMS plasma. During these periods, $\mathrm{Ar}^{+}$ constitutes $84 \%$ of the total ion flux to the substrate with $\mathrm{Ti}^{+}, \mathrm{N}_{2}{ }^{+}$, and $\mathrm{N}^{+}$ions contributing 10, 4.9, and 0.1\%. Mean $\mathrm{Ar}^{+}, \mathrm{Ti}^{+}, \mathrm{N}_{2}{ }^{+}$, and $\mathrm{N}^{+}$ion energies are 2.3, 4.8, 2.8, and $11.8 \mathrm{eV}$. The ion-to-metal flux ratio $J_{i} / J_{M e}$ measured during Ti-DCMS operation is 3.2 , essentially equal to the time-averaged value, 3.1, obtained during Al-HIPIMS/Ti-DCMS co-deposition.

The AlN concentration $x$ in as-deposited $\mathrm{Ti}_{1-x} \mathrm{Al}_{x} \mathrm{~N}$ films is $0.61 \pm 0.01$, independent of 
the substrate biasing mode. The concentration of incorporated $\mathrm{Ar}$ is 0.2 at $\%$ for $\mathrm{Ti}_{0.39} \mathrm{Al}_{0.61} \mathrm{~N}$ layers grown using continuous DC bias and less than the detection limit, 0.1 at $\%$, for the two growth modes with synchronized pulsed bias. All layers are slightly understoichiometric, with a $\mathrm{N} / \mathrm{Me}$ ratio of $0.96 \pm 0.02$. The oxygen impurity level is less than 1 at $\%$ for all three growth modes.

Significantly lower film thickness, $1.2 \mu \mathrm{m}$ for mode (1) vs. $1.9 \mu \mathrm{m}$ for modes (2) and (3), is obtained for $\mathrm{Ti}_{0.39} \mathrm{Al}_{0.61} \mathrm{~N}$ films deposited under continuous $\mathrm{DC}$ bias due to intense DCMS $\mathrm{Ar}^{+}$resputtering of the deposited film. This effect is reduced for films grown under pulsed bias.

Figure 2 shows typical $\psi$ - $2 \theta$ scans, obtained as a function of the sample tilt angle $\psi$ (defined as the angle between surface normal and the diffraction plane containing the incoming and diffracted $\mathrm{x}$-ray beams) and plotted on the same intensity scale, for $\operatorname{Ti}_{1-x} \mathrm{Al}_{x} \mathrm{~N}$ alloys grown using each of the three bias modes. $\psi$ is varied from 0 to $75^{\circ}$ in steps of $5^{\circ}$. The data are acquired in 0.1 degree steps and the dwell time per data point is $6 \mathrm{~s}$.

In all three growth modes, diffractograms are dominated by $\mathrm{NaCl}$-structure 111 and 002 film peaks. For $\mathrm{Ti}_{0.39} \mathrm{Al}_{0.61} \mathrm{~N}$ alloy films grown with continuous dc bias (see Fig. 2(a)), the relative 111 and 002 peak intensities do not vary with $\psi$. Thus, the layers are randomly oriented. In addition, the overall peak intensity is low, indicative of a small average crystallite size, consistent with TEM/XTEM results. The relaxed cubic lattice parameter $a_{o}$ obtained at the strain-free tilt angle $\psi^{*}=34.4^{\circ}$ [Ref. 7] is $4.186 \AA$, significantly higher than the expected value for single-phase cubic $\mathrm{Ti}_{1-x} \mathrm{Al}_{x} \mathrm{~N}$ films with $x=0.61, a_{o}=4.165 \AA^{7,11}$ (Density functional theory calculations yield $4.164 \AA$ ). ${ }^{12}$ This suggests that films grown in bias mode (1) are two-phase mixtures consisting of AlN-deficient $\operatorname{Ti}_{1-x} \mathrm{Al}_{x} \mathrm{~N}$ and wurtzite-structure AlN as confirmed by XTEM selected-area electron diffraction (SAED) results discussed below (see Figure 4(a)). 

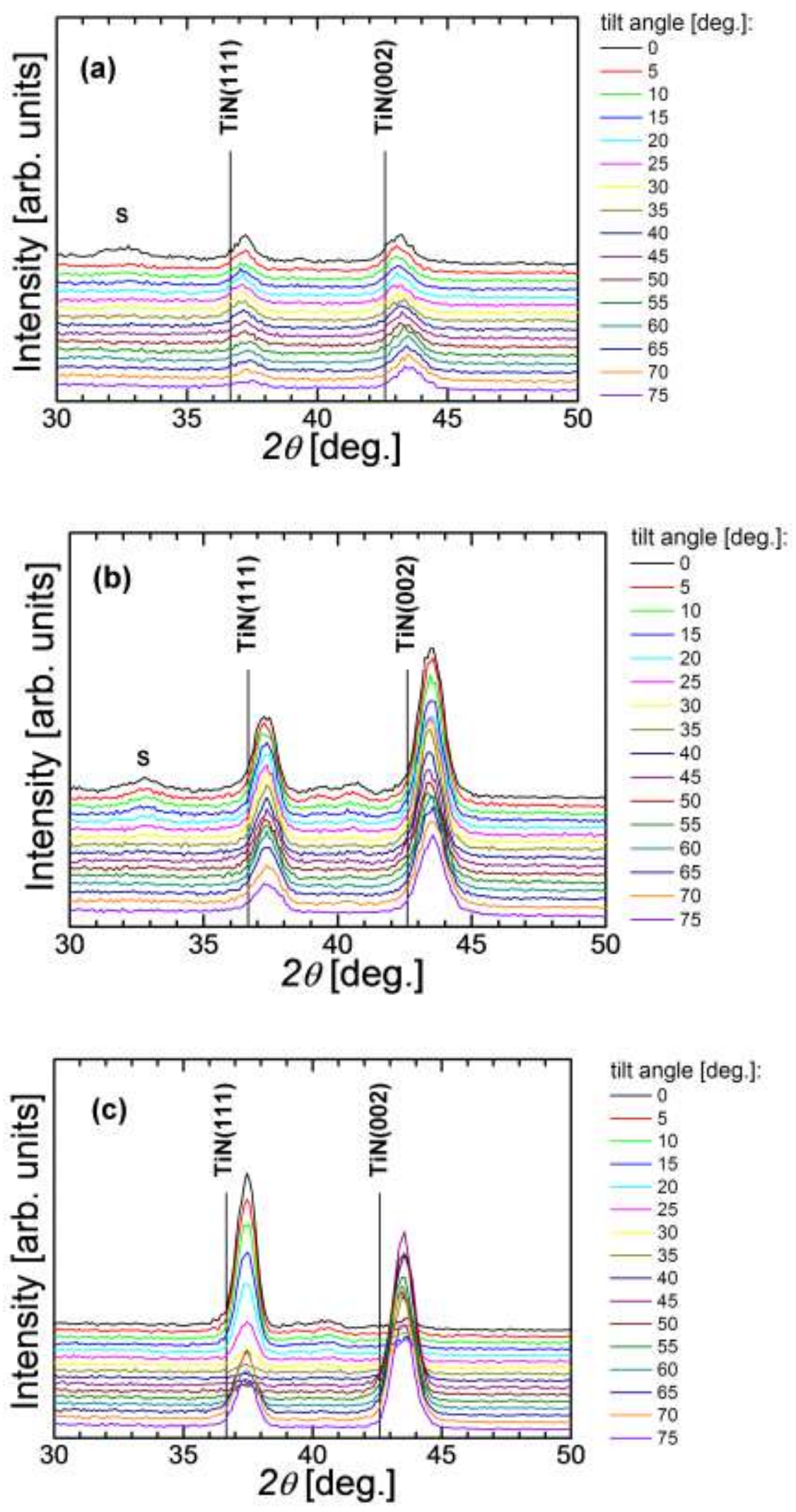

Fig.2 $\psi-2 \theta$ scans as a function of tilt angle $\psi\left(0^{\circ}\right.$ to $75^{\circ}$ from top to bottom) for $\mathrm{Ti}_{0.39} \mathrm{Al}_{0.61} \mathrm{~N}$ alloy films deposited using one of three biasing schemes: (a) continuous DCMS bias; (b) synchronous bias applied during the full $200 \mu$ s HIPIMS pulse, and (c) bias applied in synchronous with the $60 \mu$ s metal-rich-plasma portion of the HIPIMS pulse. 


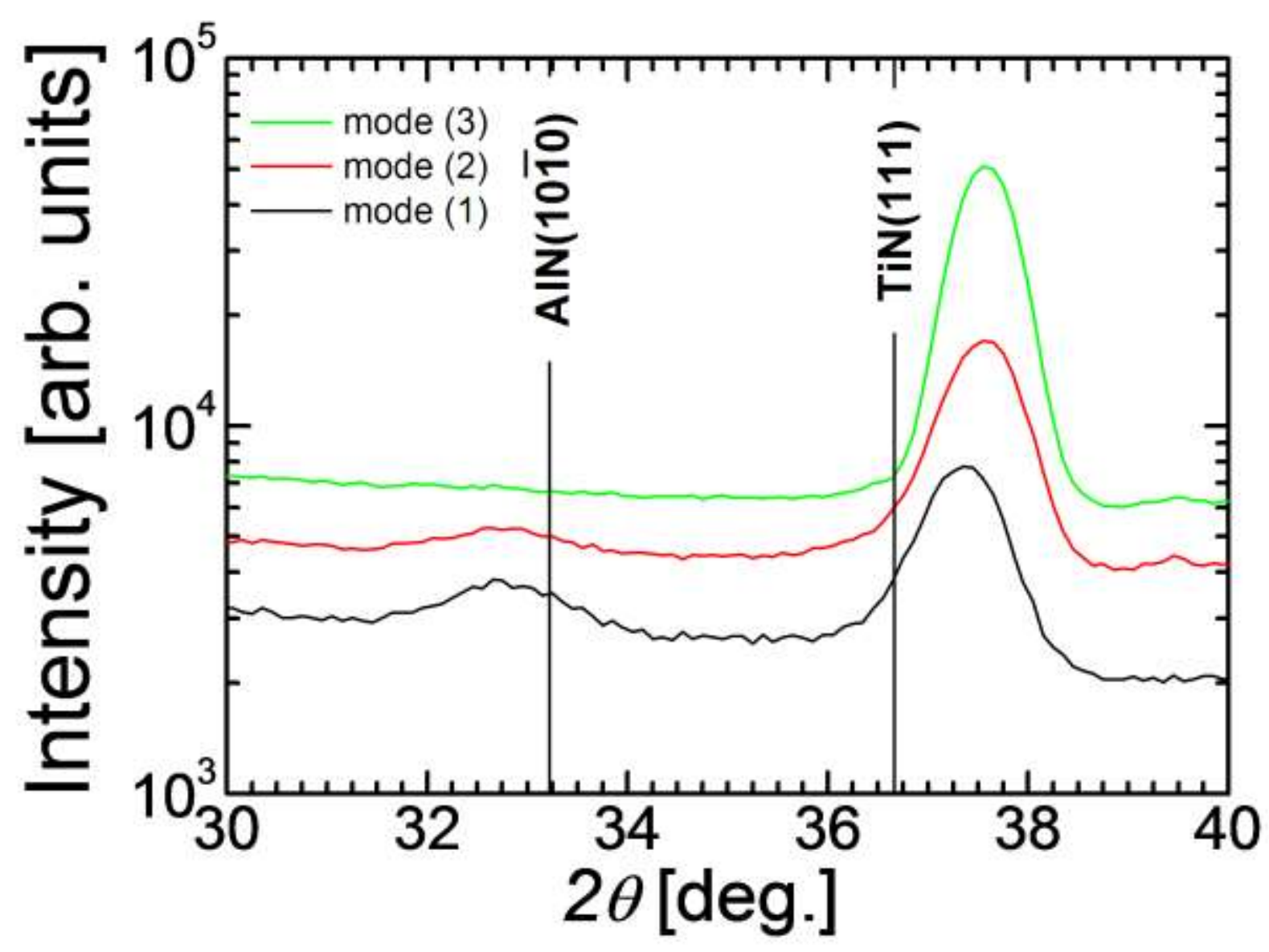

Fig.3 $\theta-2 \theta$ scans recorded at tilt angle $\psi=20^{\circ}$ for $\mathrm{Ti}_{0.39} \mathrm{Al}_{0.61} \mathrm{~N}$ films deposited using one of three biasing schemes: (a) continuous DCMS bias (mode (1), bottom); (b) synchronous bias applied during the full $200 \mu$ s HIPIMS pulse (mode (2), middle), and (c) bias applied in synchronous with the $60 \mu$ s metal-rich-plasma portion of the HIPIMS pulse (mode (3), top).

Additional confirmation of the presence of $w$-AlN in mode (1) films is obtained from XRD scans (Figure 3) carried out with $\psi=20^{\circ}$, chosen in order to minimize the Si 002 substrate peak at $2 \theta=32.96^{\circ}$ (Ref. 13) which overlaps with the $w$-AlN 1010 peak at $33.22^{\circ}$ (Ref. 14). The dwell time per data point is extended to $140 \mathrm{~s}$ in order to increase the signal-tonoise ratio. The $w$-AlN 1010 peak is observed, but shifted toward lower $2 \theta$ angles, $32.80^{\circ}$, indicating lattice expansion due to TiN incorporation into the hexagonal lattice. 
(a)
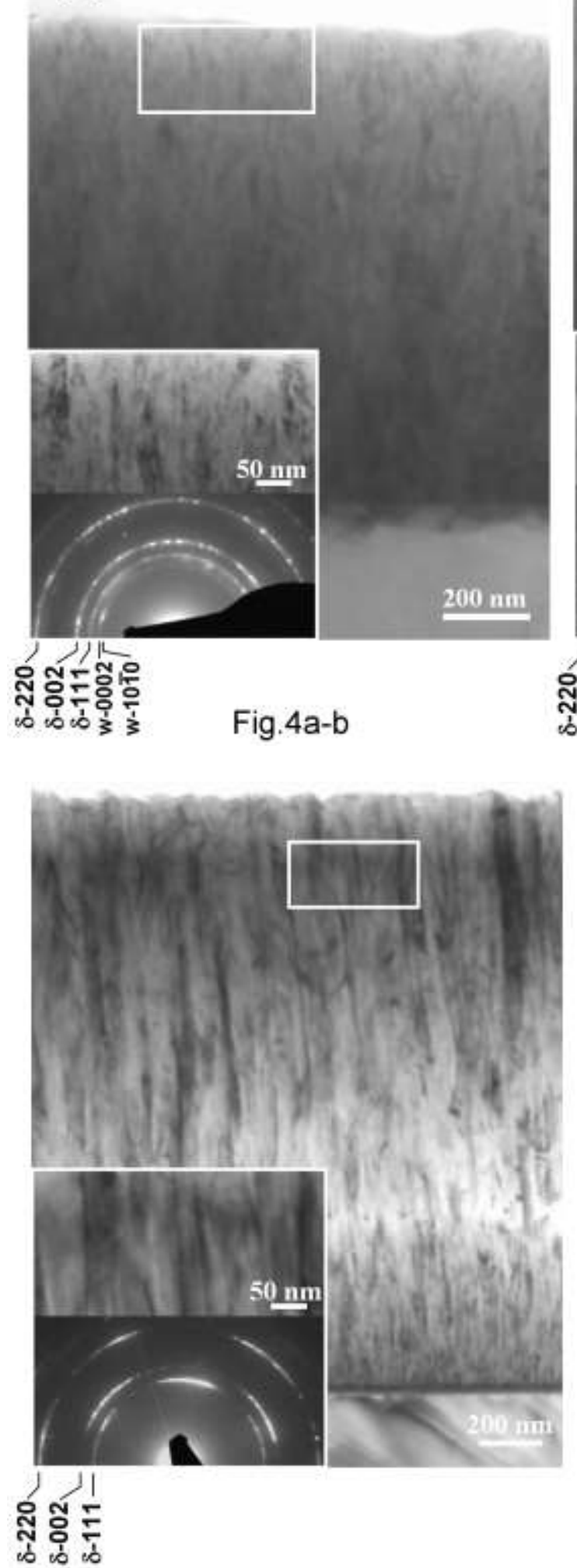

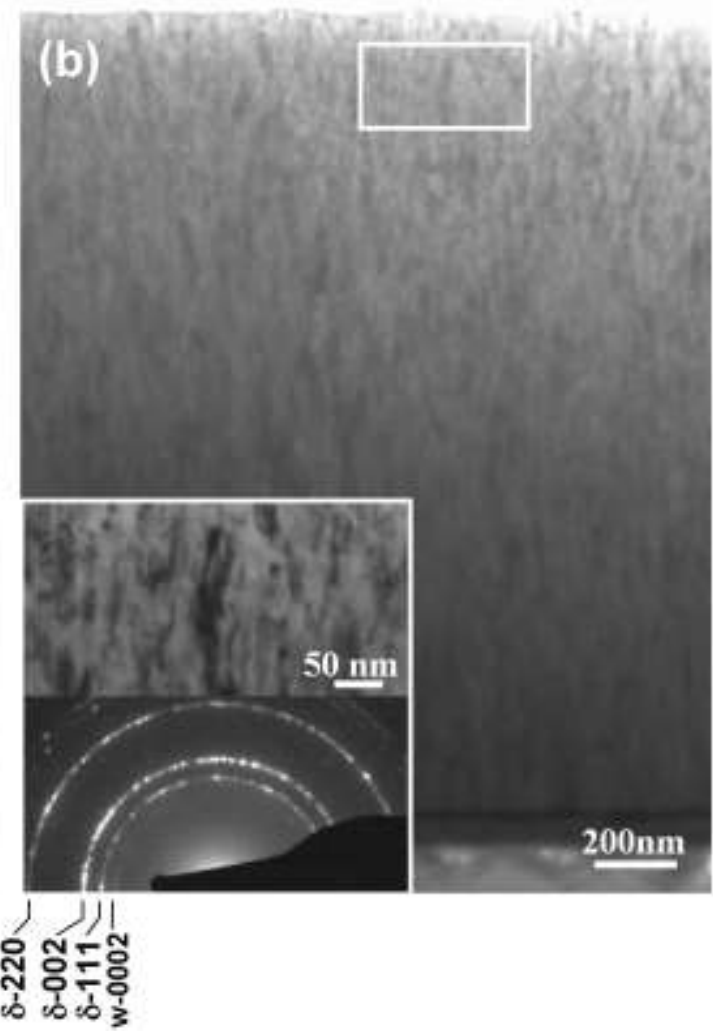

ชั่ 
Typical $\psi$-2 $\theta$ XRD scans for $\mathrm{Ti}_{0.39} \mathrm{Al}_{0.61} \mathrm{~N}$ films grown under bias mode (2) are reproduced in Fig. 2(b). Here the bias is applied only during the $200 \mu$ s Al-HIPIMS pulses, corresponding to $10 \%$ of the total deposition time. For approximately one third of each HIPIMS pulse, the ion flux incident at the film is dominated by $\mathrm{Al}^{+}$(see Fig. 1), while $\mathrm{Ar}^{+}$is the primary bombarding ion during the remainder of the pulse. There is no applied bias during pure DCMS operation between the HIPIMS pulses.

The 111 and 002 peaks in the XRD scans from bias mode (2) films have intensities at $\psi=0^{\circ}$ which are $3.4 \times$ higher than the corresponding peaks in mode (1) films, indicating a larger average grain size. The relaxed lattice parameter, $a_{o}=4.159 \AA$, is lower than for films grown under continuous dc bias and consistent with the expected value for predominately single phase $x=0.61$ alloys. $^{7,11}$ A comparison of relative peak heights as a function of $\psi$ reveals no strong preferred orientation. The corresponding $2 \theta$ scan, with $\psi=20^{\circ}$ in Fig. 3 , nevertheless reveals the presence of a small volume fraction of $w$-AlN; however, the intensity of the 1010 peak is lower than for mode (1) $\mathrm{Ti}_{0.39} \mathrm{Al}_{0.61} \mathrm{~N}$ layers. SAED patterns (see Fig. 4b) confirm the two-phase composition of mode (2) films.

Typical $\psi$-2 $\theta$ XRD scans from mode (3) films, grown under synchronous bias during metal-mode HIPIMS, are presented in Fig. 2c. The NaCl-structure 111 peak intensity is $7.2 \times$ higher than for mode (1) films and $\sim 2.4 \times$ higher than for mode (2) alloy films grown with $t=$ 0-200 $\mu$ s bias pulses. Moreover, the ratio of the relative 111/002 intensities and their evolution with increasing $\psi$ reveals that mode (3) films have a strong 111 preferred orientation. The relaxed lattice parameter is $4.153 \AA$, consistent with single-phase $x=0.61$ $\mathrm{Ti}_{1-x} \mathrm{Al}_{x} \mathrm{~N}$ alloys with a small $\mathrm{N}$ deficiency. In contrast to mode (1) and mode (2) layers, no $w$ AlN phase peak is detected in $2 \theta$ scans (Fig. 3). Mode (3) films are single-phase NaClstructure $\mathrm{Ti}_{0.39} \mathrm{Al}_{0.61} \mathrm{~N}$ alloys. 
Residual stress values $\sigma$ for alloy films grown using each of the three bias modes are obtained from $\sin ^{2} \psi$ analyses. For the randomly-oriented modes (1) and (2) films, we apply the procedure outlined in Ref. 15. Lattice spacings $d_{\psi}$ are determined for each sample from the position of the 002 TiN Bragg reflection at 16 different values of the tilt angle $\psi$ ranging from $0^{\circ}$ and $75^{\circ}$. For the highly-oriented mode (3) films, we apply $\sin ^{2} \psi$ analyses using a series of reflections belonging to the set of grains comprising the 111 fiber texture. ${ }^{16} \mathrm{We}$ measure the $111,002,022,113,133$, and $024 h k l$ peak positions at $\psi$ angles determined from the relationship

$$
\cos (\psi)=\frac{h+k+l}{\sqrt{3\left(h^{2}+k^{2}+l^{2}\right)}} .
$$

Elastic moduli $E$, required for obtaining $\sigma$ values, are acquired from nanoindentation measurements analyzed accordingly to Ref. 17. Measured $E$ values for modes (1), (2), and (3) films are 332, 427, and $377 \mathrm{GPa}$, respectively. Poisson's ratio, $v=0.19$, calculated for cubic $\mathrm{Ti}_{1-x} \mathrm{Al}_{x} \mathrm{~N}$ films with $0.5 \leq x \leq 0.75$, is obtained from Ref. 18

Mode (1) films deposited under constant bias exhibit a large compressive stress, - -3.4 GPa. For $\mathrm{Ti}_{0.39} \mathrm{Al}_{0.61} \mathrm{~N}$ layers grown using synchronized mode (2) and mode (3) bias schemes, $\sigma$ is $-0.2 \mathrm{GPa}$ and $+0.5 \mathrm{GPa}$, respectively. The measured stress values include a thermal contraction tensile stress $\sigma_{T}$ due to the samples being cooled from $T_{s}=500{ }^{\circ} \mathrm{C}$ to room temperature. $\sigma_{T}$ is given by ${ }^{19}$

$$
\sigma_{T}=\frac{E\left(\alpha_{f}-\alpha_{s}\right) \Delta T}{1-v}
$$

where $\alpha_{s}=3 \times 10^{-6} \mathrm{~K}^{-1}$ is the expansion coefficient of $\mathrm{Si}, \Delta T=477 \mathrm{~K}$, and $\alpha_{f}$ is the expansion coefficient of the alloy film, estimated as $9.35 \times 10^{-6} \mathrm{~K}^{-1} \cdot{ }^{20}$ Eqn. (2) yields $\sigma_{T}=1.2,1.6$, and 1.4 GPa for $\mathrm{Ti}_{1-x} \mathrm{Al}_{x} \mathrm{~N}$ films grown in modes (1), (2), and (3). Residual growth stresses corrected for thermal stress contributions are -4.6 for mode (1), -1.8 for mode (2), and -0.9 
GPa for mode (3) films.

Typical XTEM results are presented in Fig. 4 for films grown using each of the three bias modes. SAED patterns and higher resolution images obtained from upper regions of the films, as indicated by white rectangles, are shown as insets. Fig. 4(a) reveals that mode (1) films consist of small grains $\left(\sim 200 \times 200 \times 200-500 \AA^{3}\right)$ throughout the film thickness, elongated along the growth direction. This structure results from continuously recurring renucleation due to (i) residual damage from intense $\mathrm{Ar}^{+}$ion bombardment combined with (ii) the precipitation of incoherent $w$-AlN phase grains. Both effects interrupt local epitaxy. The corresponding SAED pattern consists of cubic $\mathrm{Ti}_{1-x} \mathrm{Al}_{x} \mathrm{~N} 111,002$, and 022, and wurtzite AlN 0002 and $10 \overline{1} 0$, diffraction rings. Uniform angular intensity of the diffracted signal is indicative of random orientation.

The nanostructure of mode (2) $\mathrm{Ti}_{0.39} \mathrm{Al}_{0.61} \mathrm{~N}$ layers, deposited using synchronized $t=$ 0-200 $\mu$ s pulsed bias (see Fig. 4(b)), is similar to that of mode (1) films, but with a larger grain size: $200-400 \AA$ in width, extending 1000-1500 $\AA$ in the growth direction. The SAED pattern reveals 111, 002, and 022 cubic diffraction rings and a very weak $w$-AlN 0002 ring. Similar to mode (1) layers, there is again no indication of preferential orientation.

A distinctly different nanostructure is observed for mode (3) films (see Fig. 4(c)) grown with the bias pulse synchronized to the metal-ion portion of the HIPIMS pulse $(t=40$ $100 \mu \mathrm{s})$. The films consist of densely-packed columns, with no open boundaries, that extend throughout the entire film thickness. A pronounced competitive columnar growth occurs initially; the average column diameter, $200 \AA$ near the substrate, increases to $600-700 \AA$ by the middle of the film, and 500-1000 ̊̊ near the film surface. Mode (3) films are single-phase $\mathrm{NaCl}$-structure with no wurtzite signal detectable by either XRD or SAED. The 111 preferred orientation determined by XRD is also evident in XTEM SAED patterns as an increased 111 intensity in the film growth direction (see the inset in Fig. 4(c)), which is tilted with respect to 
the surface normal by $\sim 20^{\circ}$ in the direction of the $\mathrm{Al}$ target.

\section{DISCUSSION}

The incident ion energy $E_{i}$ and ion-to-metal flux ratio $J_{i} / J_{M e}$ are decisive parameters controlling nanostructural evolution during low temperature $\left(<500{ }^{\circ} \mathrm{C} ; T_{s} / T_{m}<0.3\right.$ for $\left.\mathrm{TiN}\right)$ physical vapor deposition of transition metal (TM) nitrides by conventional reactive DCMS. $^{21,22,23,24,25,26,27}$ The dominant ion species incident at the growing film during DCMS with $\mathrm{N}_{2} / \mathrm{Ar}$ gas mixtures optimized to obtain stoichiometric films is typically $\mathrm{Ar}^{+}$, with $\mathrm{N}_{2}^{+}$ and $\mathrm{N}^{+}$both contributing a few percent. The $\mathrm{N}_{2}{ }^{+} / \mathrm{N}^{+}$ratio increases with increasing $\mathrm{N}_{2} / \mathrm{Ar}$ fraction, while in pure $\mathrm{N}_{2}$ discharges the dominant ion species is $\mathrm{N}_{2}{ }^{+28}$ For magnetron sputtering, in which anode sheaths are of the order of $\sim 1 \mathrm{~mm}$, the ion energy $E_{i}$ incident at the growing film corresponds closely to the applied negative substrate bias $V_{s}{ }^{21,22}$ That is, $E_{i}=$ $e\left(V_{s}-V_{p l}\right)$, where the plasma potential $V_{p l}$ is typically $\sim 3-5 \mathrm{~V} \cdot{ }^{29}$

Three polycrystalline TM nitride ion-assisted growth regimes have been identified. ${ }^{19-25,28}$ (i) Under weak ion irradiation conditions $\left(J_{i} / J_{M e} \square 1 ; E_{i} \square 20 \mathrm{eV}\right.$ ) with low ion flux and ion energy, $E_{i}$ is maintained below the bulk lattice atom displacement threshold. Film microstructure develops toward underdense columnar 111 texture via kinetically-limited competitive growth; adatoms have higher residence times on low-chemical-potential, low-diffusivity 111 planes. (ii) Increasing $J_{i} / J_{M e}$ to $\sim 5-10$ while maintaining $E_{i} \square 20 \mathrm{eV}$, results in film densification. However, growth in regime (ii) remains competitive, but preferred orientation now evolves toward 002, rather than 111, texture. This is due to higher $\mathrm{N}$ coverage $\theta_{N}$ on 001 grains, resulting from $\mathrm{N}^{+}$adsorption and $\mathrm{N}_{2}{ }^{+}$collisionally-induced dissociative chemisorption, dramatically decreasing metal cation mean-free paths, nucleation lengths $L_{002}$, and the 002 chemical potential $\mu_{002}{ }^{25} 111$ is a polar direction in NaCl-structure 
nitrides for which the surface is always $\mathrm{N}$-terminated; thus, the $\theta_{N}, L_{111}$, and the chemical potential $\mu_{111}$ remain constant. ${ }^{26}$ The resulting switch in chemical potential values, such that $\mu_{002}$ becomes less than $\mu_{111}$, leads to the observed transformation in texture which now evolves to 002. Films grown in regimes (i) and (ii) exhibit no significant compressive stress.

A third regime (iii) of ion-assisted growth, which is widely utilized in conventional DC bias sputter deposition, is characterized by low ion densities, $J_{i} / J_{M e} \square 1$, combined with relatively high ion energies, $E_{i} \square 50 \mathrm{eV}$, sufficient to generate linear cascade effects including recoil implantation and forward sputtering. This, in turn, leads to significant concentrations of trapped residual defects and a correspondingly high compressive stress. Dense 002 structures also develop under regime (iii). However, the mechanism for 002 texture formation is quite different than in regime (ii). In regime (iii), orientation evolves toward those planes which are least dense. Ion energy in open channel directions is distributed over larger depths yielding less lattice distortion and lower sputter yields. Therefore, planes orthogonal to open channel directions have higher survival probability. For film growth in this ion-assisted regime, several research groups have shown that the product of $E_{i}$ and $J_{i} / J_{M e}$-- the average energy $\left(E_{d}\right)$ per deposited atom -- can be correlated with film properties. ${ }^{15,30,31,32}$

Studies of nanostructural evolution and the phase stability of metastable $\mathrm{Ti}_{0.5} \mathrm{Al}_{0.5} \mathrm{~N}$ alloy films grown in regime (iii) have shown that with $E_{d}>100 \mathrm{eV} / \mathrm{atom}$, ion irradiation results in precipitation of second-phase hexagonal $\mathrm{AlN}$ grains leading to $\operatorname{Ti}_{1-x} \mathrm{Al}_{x} \mathrm{~N}$ renucleation and a fine-grain two-phase nanostructure. ${ }^{33}$ In addition to $E_{i}$ and $J_{i} / J_{M e}$, momentum transfer to the growing film surface, controlled by the combination of ion/film atomic mass match and ion energy, as well as the chemical nature of the ion, inert gas $v s$. metal, are also expected to play an important role in controlling structural evolution. We focus particularly on these latter effects in the present experiments. 
For $\mathrm{Ti}_{0.39} \mathrm{Al}_{0.61} \mathrm{~N}$ growth by Al-HIPIMS/Ti-DCMS deposition, the average energy per deposited atom delivered to the film growth surface, including that provided by the DCMS plasma, during an entire $\tau=2 \mathrm{~ms}$ period, is

$$
\left\langle\bar{E}_{d}\right\rangle=\frac{1}{\tau} \int_{0}^{\tau} E_{i}(t)\left(J_{i} / J_{M e}\right)(t) d t
$$

In our experiments, $J_{i} / J_{M e}(\mathrm{HIPIMS}) \sim J_{i} / J_{M e}(\mathrm{DCMS}) \sim 3.1$; hence, $\left\langle\bar{E}_{d}\right\rangle$ is primarily determined by the time-averaged energy of incident ions, $\left\langle E_{i}\right\rangle=\frac{1}{\tau} \int_{0}^{\tau} E_{i}(t) d t$.

The substrate bias, $V_{s}=-60 \mathrm{~V}$, is applied during $100 \%$ of the deposition time (mode 1), $10 \%$ (mode 2), and 3\% (mode 3). For the remaining deposition time, in modes (2) and (3), the growing films are at floating potential $V_{f} \sim-10 \mathrm{~V}$. Using the mean ion energies and relative contributions to the total ion flux obtained from the in-situ ion mass spectrometry analyses, $\left\langle\bar{E}_{d}\right\rangle=\left\langle E_{i}\right\rangle \times J_{i} / J_{M e}$ is approximately $200 \mathrm{eV} /$ atom for continuous DC bias (mode 1), $55 \mathrm{eV} /$ atom for the full $200 \mu$ s HIPIMS pulse (mode 2), and $45 \mathrm{eV} /$ atom in the case for which the bias pulse is synchronized with the metal-rich portion of the high-power pulse (mode 3).

During mode (1) growth, ion irradiation with $\left\langle\bar{E}_{d}\right\rangle=200 \mathrm{eV} /$ atom results in the precipitation of a significant mole fraction of second-phase $w$-AlN, as evident from both XRD (Fig. 3) and SAED results (Fig. 4(a)). Intense uninterrupted $\mathrm{Ar}^{+}$ion bombardment, with incident fluxes $3 \times$ that of the deposited metal, leads to large transient, and smaller residual, defect concentrations. The transient defect flux derives from local microscopic compressive stress driving implanted Ar and excess recoil-implanted lattice atoms toward the surface in order to partially relax the lattice. 
The combination of transient and the residual trapped defects destabilizes the metastable $\mathrm{NaCl}$ structure and facilitates the precipitation of second phase $w$-AlN. This, in turn, disrupts local epitaxial growth on cubic $\mathrm{NaCl}$-structure grains. The resulting continuous renucleation gives rise to the formation of a randomly-oriented, thickness-invariant, finegrain $\left(\sim 200 \times 200 \times 200-500 \AA^{3}\right)$ nanostructure, with grains slightly elongated in the growth direction. The intense $\mathrm{Ar}^{+}$ion irradiation during mode (1) growth also results in extensive material loss by resputtering, predominantly during the DCMS phase, leading to thinner films for a fixed deposition time. The presence of residual trapped Ar, 0.2 at $\%$, and ion-induced defects explain the observed high compressive stress, $-4.6 \mathrm{GPa}$.

$\left\langle\bar{E}_{d}\right\rangle$ is reduced to $55 \mathrm{eV} /$ atom during mode (2) growth; thus, there is less resputtering and thicker films are obtained. The correspondingly lower defect densities result in less renucleation; hence, the average grain size is larger $\left(\sim 300 \times 300 \times 1000-1500 \AA^{3}\right)$, with grains more elongated in the growth direction, and the volume fraction of $w$-AlN second phase precipitates is lower. The incorporated Ar concentration is below detection limits and the residual stress is significantly decreased to $-1.8 \mathrm{GPa}$.

In mode (3) film growth, for which $\left\langle\bar{E}_{d}\right\rangle=45 \mathrm{eV} /$ atom, $\mathrm{Ti}_{0.39} \mathrm{Al}_{0.61} \mathrm{~N}$ layers are singlephase, local epitaxy is uninterrupted, and the films exhibit a columnar nanostructure (see Fig.4(c)) in which the average column width $w$ increases with film thickness $h$. During the early stages of mode (3) growth, films exhibit a randomly oriented, fine-grain equiaxed structure $\left(\sim 200 \times 200 \times 200 \mathrm{~A}^{3}\right)$, from which, unlike layers grown in modes (1) and (2), a kinetically-limited 111 texture slowly evolves. The average column width $w$ increases with film thickness $h$, giving rise to V-shaped columns with diameters of 500-1000 $\AA$ at the surface, $h=1.9 \mu \mathrm{m}$. The 111 texture emerges due to larger adatom residence times on low-potential-energy 111 , than 002 , surfaces. ${ }^{22,26}$ Residual defect concentrations, and hence 
residual stress $(-0.9 \mathrm{GPa})$, is lower under $\mathrm{Al}^{+}$, rather than $\mathrm{Ar}^{+}$, ion irradiation since $\mathrm{Al}$ is primarily incorporated into the lattice of the growing film, whereas Ar resides in interstitial sites resulting in compressive stress. The 111 columnar structure is fully dense, with no observable intra- and intercolumnar voids. Thus, $\mathrm{Al}^{+}$irradiation with $\left\langle\bar{E}_{d}\right\rangle=45 \mathrm{eV} / \mathrm{atom}$ and $J_{i} / J_{M e} \sim 3$ results in sufficient lattice atom displacements (through recoil implantation and forward sputtering) to eliminate the voids typically observed in low-temperature $\left(T_{s} / T_{m} \leq\right.$ $0.3)$ TM nitride films..$^{21,23,24,26,27}$

The relatively small decrease in $\left\langle\bar{E}_{d}\right\rangle$ from $55 \mathrm{eV} /$ atom for mode (2) to $45 \mathrm{eV} /$ atom for mode (3) films can not entirely explain the dramatic nanostructural differences observed in the two cases. Instead, we attribute most of the difference to the fact that the majority of energetic ions incident at the growing film surface are $\mathrm{Al}^{+}$during mode (3), vs. $\mathrm{Ar}^{+}$ions in mode (2) growth, due to substrate bias synchronization with the metallic portion of the HIPIMS pulses. There are at least two reasons which can be proposed for this: differences in momentum transfer to the growing film and, as noted above, the fact that $\mathrm{Al}$ is incorporated in lattice sites, while Ar is incorporated as interstitials.

Momentum transfer $\Phi$ between an incident ion with mass $m_{i}$ and the film with average mass $m_{f}$ is given by $\Phi=4 m_{f} m_{i} /\left(m_{f}+m_{i}\right)^{2}$ for $180^{\circ}$ hard-sphere collisions. Here, $m_{i}=26.98$ amu and $39.95 \mathrm{amu}$ for $\mathrm{Al}^{+}$and $\mathrm{Ar}^{+}$, while $m_{f}=24.5 \mathrm{amu}$. Thus, $\Phi=0.998$ for $\mathrm{Al}^{+}$irradiation and 0.944 for $\mathrm{Ar}^{+}$bombardment. ${ }^{34}$ This difference is too small to account for the dramatic change in nanostructure observed between modes (2) and (3) films.

Thus, the primary difference in nanostructural evolution results from the chemical nature of the ions - inert gas $v s$. constituent metal ions. With $J_{i} / J_{M e} \sim 3$ and $E_{i} \square 70 \mathrm{eV}$, there is a large dynamic concentration of implanted near-surface Ar during mode (2) $\operatorname{Ti}_{0.39} \mathrm{Al}_{0.61} \mathrm{~N}$ growth. Most of the implanted Ar diffuses back to the surface, driven by compressive strain, 
with a migration rate enhanced by high ion-induced near-surface defect concentrations. Thus, there are continuous opposing Ar implantation and out-diffusion fluxes during film growth. This high transient defect concentration, together with the trapped residual defects, destabilizes the metastable $\mathrm{NaCl}$-structure host lattice resulting in second-phase $w$-AlN precipitation, loss of local epitaxy, and the formation of the fine-grained randomly-oriented nanostructure observed in both modes (1) and (2) films.

Energetic $\mathrm{Al}^{+}$ions cause sufficient lattice-atom displacements to eliminate film porosity. However, Al, as opposed to Ar, is primarily incorporated in lattice sites, as indicated by the low stress, and does not result in nucleation of $w$-AlN. Mode (3) films have a dense, columnar, 111 oriented microstructure which is not attainable in regimes (i)-(iii) of standard bias magnetron reactive sputtering for which the structure varies from underdense 111 to dense 002 .

Applying a pulsed bias synchronized with the metal-rich plasma opens a new pathway for microstructure evolution and control which is a combination of regimes (i) and (iii). The (111) texture evolves through kinetically-limited competitive growth following the development of a fine-grained randomly-oriented structure during the nucleation, coalescence, and early stages of film growth. The 111 columns, whose average widths increase with film thickness, slowly win the growth competition due to higher adatom residence times on low-chemical-potential, low-diffusivity 111 planes. Similar to growth regime (iii), the bombarding $\mathrm{Al}^{+}$ions have sufficient energy to penetrate the lattice and cause densification via forward momentum transfer in shallow overlapping cascades. However, $\mathrm{Al}^{+}$ ions are primarily incorporated at lattice sites, do not result in high residual defect concentrations, and thus preserve local 111 epitaxial growth.

\section{CONCLUSIONS}


In summary, we demonstrate that the use of a synchronous pulsed bias during hybrid HIPIMS/DCMS co-sputter deposition of $\mathrm{Ti}_{1-x} \mathrm{Al}_{x} \mathrm{~N}$ decreases, or eliminates, depending on whether the bias is applied during the entire HIPIMS pulse or only during the metal ion phase, the compressive stress associated with trapped rare gas. Alloy films grown with continuous dc bias are small-grain, two-phase mixtures, consisting of AlN-deficient NaClstructure $\mathrm{Ti}_{1-x} \mathrm{Al}_{x} \mathrm{~N}$ and wurtzite-structure $\mathrm{AlN}$, with high compressive stress, a large concentration of trapped Ar, and no preferred crystallographic orientation. Synchronizing the bias with the full HIPIMS pulse to the Al target results in films which have significantly lower compressive stress with no measureable Ar incorporation, larger average grain size, a smaller volume fraction of wurtzite AlN, and random texture. In contrast, by synchronizing the bias with the metal plasma phase of the HIPIMS pulses, high-energy $\mathrm{Ar}^{+}$ion irradiation is greatly reduced in favor of irradiation predominantly by $\mathrm{Al}^{+}$ions. The resulting single-phase

$\mathrm{NaCl}$-structure $\mathrm{Ti}_{0.39} \mathrm{Al}_{0.61} \mathrm{~N}$ alloys have a dense columnar structure with a strong 111 orientation and are nearly stress-free.

\section{ACKNOWLEDGEMENTS}

The financial support from the Swedish VINN Excellence Center on Functional Nanoscale Materials (FunMat) is acknowledged. We thank the staff at the Tandem Laboratory, Uppsala University, for technical support. 
${ }^{1}$ D. McIntyre, J.E. Greene, G. Håkansson, J.-E. Sundgren, W.-D. Munz Oxidation of Metastable Single-phase Polycrystalline Ti $i_{0.5} A l_{0.5} N$ Films: Kinetics and Mechanisms J. Appl. Phys. 67, 1542-53 (1990).

${ }^{2}$ O. Knotek, M. Böhmer, T. Leyendecker On Structure and Properties of Sputtered Ti and Al Based Hard Compound Films J. Vac. Sci. Technol. A 4, 2695 (1986).

${ }^{3}$ F. Adibi, I. Petrov, L. Hultman, U. Wahlström, T. Shimizu, D. McIntyre, J.E. Greene J. Appl. Phys. 69, 6437 (1991).

${ }^{4}$ J.S. Schuster and J. Bauer, J. Solid State Chem. 53, 260 (1984).

${ }^{5}$ H. Holleck, Surf. Coat. Tech. 36, 151 (1988).

${ }^{6}$ G. Greczynski, J. Lu, M. Johansson, J. Jensen, I. Petrov, J.E. Greene, and L. Hultman Selection of Metal Ion Irradiation for Controlling $T i_{1-x} A l_{x} N$ Alloy Growth via Hybrid HIPIMS/magnetron Co-sputtering, Vacuum 86, 1036 (2012).

${ }^{7}$ G. Greczynski, J. Lu, M. Johansson, J. Jensen, I. Petrov, J.E. Greene, and L. Hultman, Role of Ti ${ }^{n+}$ and $A l^{n+}$ Ion Irradiation $(n=1,2)$ During $T i_{I_{-x}} A l_{x} N$ Alloy Film Growth in a Hybrid HIPIMS/magnetron Mode, Surf. Coat. Tech. 206, 4202 (2012).

${ }^{8}$ http://www.cemecon.de/coating_technology/2_coating_units/25_cc_800sup_sup_9_hipims/index_eng.html, accessed in September, 2011.

${ }^{9}$ See, for example, Chapter 6 in M. Birkholz Thin Film Analysis by X-ray Scattering, Wiley-VCH, Weinheim (2006).

${ }^{10}$ David R. Lide (ed), CRC Handbook of Chemistry and Physics, 84th Edition. CRC Press. Boca Raton, Florida, 2003; Section 10, Atomic, Molecular, and Optical Physics; Ionization Potentials of Atoms and Atomic Ions.

${ }^{11}$ F. Adibi, I. Petrov, J. E. Greene, U. Wahlström, and J.-E. Sundgren, Design and Characterization of a Compact Two-Target Ultrahigh Vacuum Magnetron Sputter Deposition System: Application to the Growth of Epitaxial $T i_{I_{-x}} A l_{x} N$ alloys and TiN/Ti $i_{1-x} A l_{x} N$ Superlattices, J. Vac. Sci. Technol. A, 11, 136 (1993).

${ }^{12}$ B. Alling, A. Karimi, I.A. Abrikosov Electronic Origin of the Isostructural Decomposition in Cubic $M_{1-x} A l_{x} N$ (M=Ti, Cr, Sc, Hf): A First-principles Study Surf. Coat. Tech. 203, 883 (2008).

${ }^{13}$ Although the $\mathrm{Si} 002$ reflection is forbidden by extinction rules, it can appear due to multiple scattering.

${ }^{14}$ The JCPDS database (1998), card: 25-1133; Kohn et al. Am. Mineral 41, 355 (1956).

${ }^{15}$ J. Musil, S. Kadlec, V. Valvoda, R. Kuzel, and R. Cerny, Surf. Coat. Tech. 43/44, 259 (1990).

${ }^{16}$ Y.-W. Kim, J. Moser, I. Petrov, J. E. Greene, and S. M. Rossnagel, Directed Sputter Deposition of AlCu: Film Microstructure and Microchemistry, J. Vac. Sci. Technol. A, 12, 3169 (1994).

${ }^{17}$ W. C. Oliver and G. M. Pharr, J. Mater. Res. 7, 1564 (1992).

${ }^{18}$ V. Podgursky Ab initio Calculations of Elastic Properties of Isotropic and Oriented $T_{i_{1}-x} A l_{x} N$ Hard Coatings J. Phys. D: Appl. Phys. 40, 4021 (2007).

${ }^{19}$ See, for example, M. Ohring, Materials Science of Thin Films, $2^{\text {nd }}$ edition, Academic Press, 2001, p. 732-733.

${ }^{20}$ Here, we use $\alpha_{f}$ for TiN since there are no published values for $\mathrm{Ti}_{0.39} \mathrm{Al}_{0.61} \mathrm{~N}$. We expect $\alpha_{f}$ to be larger for the alloy; hence, $\sigma_{T}$ values obtained from eqn. (2) are lower limits.

${ }^{21}$ I. Petrov, F. Adibi, J. E. Greene, L. Hultman and J.-E. Sundgren, Average Energy Deposited per Atom: A Universal Parameter for Describing Ion-Assisted Film Growth?, Appl. Phys. Lett. 63, 36 (1993).

${ }^{22}$ J. E. Greene, J.-E. Sundgren, L. Hultman, I. Petrov, and D. B. Bergstrom, Development of Preferred Orientation in Polycrystalline TiN Layers Grown by Ultra-High-Vacuum Reactive Magnetron Sputtering, Appl. Phys. Lett. 67, 2928 (1995).

${ }^{23}$ I. Petrov, P.B. Barna, L. Hultman, J.E. Greene Microstructural Evolution During Film Growth J. Vac. Sci. Technol. A 21, 117 (2003).

${ }^{24}$ C.-S. Shin, Y.-W. Kim, D. Gall, J. E. Greene, and I. Petrov, Phase Composition and Microstructure of Polycrystalline and Epitaxial TaN ${ }_{x}$ Layers Grown on oxidized Si(001) and $\mathrm{MgO}(001)$ by Reactive Magnetron Sputter Deposition, Thin Solid Films 402, 172 (2002).

${ }^{25}$ D. Gall, C.-S. Shin, T. Spila, M. Odén, M. J. H. Senna, J. E. Greene, and I. Petrov, Growth of Single-crystal CrN on MgO(001): Effects of Low-energy Ion-irradiation on Surface Morphological Evolution and Physical Properties J. Appl. Phys. 91, 3589 (2002).

${ }^{26}$ D. Gall, I. Petrov, N. Hellgren, L. Hultman, J.-E. Sundgren, and J. E. Greene, Growth of Poly- and SingleCrystal ScN on $\mathrm{MgO}(001)$ : Role of Low-Energy $\mathrm{N}_{2}^{+}$Irradiation in Determining Texture, Microstructure Evolution, and Mechanical Properties, J. Appl. Phys. 84, 6034 (1998).

${ }^{27}$ D. Gall, I. Petrov, and J. E. Greene, Epitaxial Sc1-xTixN(001: Optical and Electronic Transport Properties, J. Appl. Phys., 89, 401 (2001). 
${ }^{28}$ I. Petrov, A. Myers, J. E. Greene, and J. R. Abelson, Mass and Energy Resolved Detection of Ions and Neutral Sputtered Species Incident at the Substrate during Reactive Magnetron Sputtering of Ti in Mixed Ar+N2 Discharges, J. Vac. Sci. Technol. A, 12, 2846 (1994).

${ }^{29}$ I. Petrov, F. Adibi, J. E. Greene, W. D. Sproul and W.-D. Münz, Use of an Externally Applied Axial Magnetic Field to Control Ion/Neutral Flux Ratios Incident at the Substrate During Magnetron Sputter Deposition, J. Vac. Sci. Technol. A, 10, 3283 (1992).

${ }^{30}$ J. M. E. Harper, J. J. Cuomo, R. J. Gambino, and H. R. Kaufman, in Ion Bombardment Modification of Surfaces, edited by 0. Auciello and R. Kelly (Elsevier, New York, 1984). Chap. 4.

${ }^{31}$ T. C. Huang, G. Lim, F. Parmigiani, and E. Kay, J. Vac. Sci. Technol. A 3, 2161 (1985).

32 J. E. Yehoda, B. Yang, K. Vedam, and R. Messier, J. Vac. Sci. Technol. A 6, 1631 (1988).

${ }^{33}$ F. Adibi, I. Petrov, J. E. Greene, L. Hultman, and J.-E. Sundgren, Effects of High Flux Low-Energy $(<50 \mathrm{eV})$ Ion Irradiation During Deposition on the Microstructure and Preferred Orientaion of $T i_{0.5} A l_{0.5} N$ Alloys Grown by UHV Reactive Magnetron Sputtering, J. Appl. Phys. 73, 8580 (1993).

${ }^{34}$ The values for energetic $\mathrm{Ar}^{+}$and $\mathrm{Al}^{+}$impinging on $\mathrm{Ti}, \mathrm{Al}$, and $\mathrm{N}$ are $0.992,0.962$, and 0.768 , and $0.920,1$, and 0.899 , respectively. 